\title{
Boundary Blow-Up Solutions to $p(x)$-Laplacian Equations with Exponential Nonlinearities
}

\author{
Qihu Zhang \\ Department of Mathematics and Information Science, Zhengzhou University of Light Industry, \\ Zhengzhou, Henan 450002, China
}

Correspondence should be addressed to Qihu Zhang, zhangqh1999@yahoo.com.cn

Received 18 August 2007; Accepted 25 November 2007

Recommended by M. Garcia-Huidobro

This paper investigates the $p(x)$-Laplacian equations with exponential nonlinearities $-\Delta_{p(x)} u$ $+e^{f(x, u)}=0$ in $\Omega, u(x) \rightarrow+\infty$ as $d(x, \partial \Omega) \rightarrow 0$, where $-\Delta_{p(x)} u=-\operatorname{div}\left(|\nabla u|^{p(x)-2} \nabla u\right)$ is called $p(x)$ Laplacian. The singularity of boundary blow-up solutions is discussed, and the existence of boundary blow-up solutions is given.

Copyright (0) 2008 Qihu Zhang. This is an open access article distributed under the Creative Commons Attribution License, which permits unrestricted use, distribution, and reproduction in any medium, provided the original work is properly cited.

\section{Introduction}

The study of differential equations and variational problems with nonstandard $p(x)$-growth conditions is a new and interesting topic. We refer to [1,2], the background of these problems. Many results have been obtained on this kind of problems, for example, [1-15]. In this paper, we consider the $p(x)$-Laplacian equations with exponential nonlinearities

$$
\begin{gathered}
-\Delta_{p(x)} u+e^{f(x, u)}=0 \quad \text { in } \Omega, \\
u(x) \longrightarrow+\infty \quad \text { as } d(x, \partial \Omega) \longrightarrow 0,
\end{gathered}
$$

where $-\Delta_{p(x)} u=-\operatorname{div}\left(|\nabla u|^{p(x)-2} \nabla u\right), \Omega=B(0, R) \subset \mathbb{R}^{N}$ is a bounded radial domain $(B(0, R)=$ $\left.\left\{x \in \mathbb{R}^{N}|| x \mid<R\right\}\right)$. Our aim is to give the existence and asymptotic behavior of solutions for problem $(\mathrm{P})$.

Throughout the paper, we assume that $p(x)$ and $f(x, u)$ satisfy that

$\left(\mathrm{H}_{1}\right) p(x) \in C^{1}(\bar{\Omega})$ is radial and satisfies

$$
1<p^{-} \leq p^{+}<+\infty, \quad \text { where } p^{-}=\inf _{\Omega} p(x), p^{+}=\sup _{\Omega} p(x) ;
$$


$\left(\mathrm{H}_{2}\right) f(x, u)$ is radial with respect to $x, f(x, \cdot)$ is increasing and $f(x, 0)=0$ for any $x \in \Omega$;

$\left(\mathrm{H}_{3}\right) f: \Omega \times \mathbb{R} \rightarrow \mathbb{R}$ is a continuous function and satisfies

$$
|f(x, t)| \leq C_{1}+C_{2}|t|^{\gamma(x)}, \quad \forall(x, t) \in \Omega \times \mathbb{R},
$$

where $C_{1}, C_{2}$ are positive constants, $0 \leq \gamma \in C(\bar{\Omega})$.

The operator $-\Delta_{p(x)} u=-\operatorname{div}\left(|\nabla u|^{p(x)-2} \nabla u\right)$ is called $p(x)$-Laplacian. Especially, if $p(x) \equiv$ $p$ (a constant), (P) is the well-known $p$-Laplacian problem (see [16-18]).

Because of the nonhomogeneity of $p(x)$-Laplacian, $p(x)$-Laplacian problems are more complicated than those of $p$-Laplacian ones (see [6]); and another difficulty of this paper is that $f(x, u)$ cannot be represented as $h(x) f(u)$.

\section{Preliminary}

In order to deal with $p(x)$-Laplacian problems, we need some theories on spaces $L^{p(x)}(\Omega)$ and $W^{1, p(x)}(\Omega)$, and properties of $p(x)$-Laplacian, which we will use later (see $\left.[3,7]\right)$. Let

$$
L^{p(x)}(\Omega)=\left\{u \mid u \text { is a measurable real-valued function, } \int_{\Omega}|u(x)|^{p(x)} d x<\infty\right\} .
$$

We can introduce the norm on $L^{p(x)}(\Omega)$ by

$$
|u|_{p(x)}=\inf \left\{\lambda>\left.0\left|\int_{\Omega}\right| \frac{u(x)}{\lambda}\right|^{p(x)} d x \leq 1\right\} .
$$

The space $\left(L^{p(x)}(\Omega),|\cdot|_{p(x)}\right)$ becomes a Banach space. We call it generalized Lebesgue space. The space $\left(L^{p(x)}(\Omega),|\cdot|_{p(x)}\right)$ is a separable, reflexive, and uniform convex Banach space (see [3, Theorems 1.10, 1.14]).

The space $W^{1, p(x)}(\Omega)$ is defined by

$$
W^{1, p(x)}(\Omega)=\left\{u \in L^{p(x)}(\Omega)|| \nabla u \mid \in L^{p(x)}(\Omega)\right\},
$$

and it can be equipped with the norm

$$
\|u\|=|u|_{p(x)}+|\nabla u|_{p(x)^{\prime}} \quad \forall u \in W^{1, p(x)}(\Omega) .
$$

$W_{0}^{1, p(x)}(\Omega)$ is the closure of $C_{0}^{\infty}(\Omega)$ in $W^{1, p(x)}(\Omega) . W^{1, p(x)}(\Omega)$ and $W_{0}^{1, p(x)}(\Omega)$ are separable, reflexive, and uniform convex Banach spaces (see [3, Theorem 2.1]).

If $u \in W_{\text {loc }}^{1, p(x)}(\Omega) \cap C(\Omega), u$ is called a solution of $(\mathrm{P})$ if it satisfies

$$
\int_{Q}|\nabla u|^{p(x)-2} \nabla u \nabla q d x+\int_{Q} f(x, u) q d x=0, \quad \forall q \in W_{0}^{1, p(x)}(Q),
$$

for any domain $Q \Subset \Omega$, and $\max (k-u, 0) \in W_{0}^{1, p(x)}(\Omega)$ for any $k \in \mathbb{N}^{+}$.

Let $W_{0, \text { loc }}^{1, p(x)}(\Omega)=\left\{u \mid\right.$ there exists an open domain $Q \Subset \Omega$ s.t. $\left.u \in W_{0}^{1, p(x)}(Q)\right\}$. For any $u \in W_{\mathrm{loc}}^{1, p(x)}(\Omega) \cap C(\Omega)$ and $\varphi \in W_{0, \mathrm{loc}}^{1, p(x)}(\Omega)$, define $A: W_{\mathrm{loc}}^{1, p(x)}(\Omega) \cap C(\Omega) \rightarrow\left(W_{0, \mathrm{loc}}^{1, p(x)}(\Omega)\right)^{*}$ as $\langle A u, \varphi\rangle=\int_{\Omega}\left(|\nabla u|^{p(x)-2} \nabla u \nabla \varphi+e^{f(x, u)} \varphi\right) d x$. 
Lemma 2.1 (see $\left[5\right.$, Theorem 3.1]). Let $h \in W^{1, p(x)}(\Omega) \cap C(\Omega), X=h+W_{0, \mathrm{loc}}^{1, p(x)}(\Omega) \cap C(\Omega)$. Then, $A: X \rightarrow\left(W_{0, l o c}^{1, p(x)}(\Omega)\right)^{*}$ is strictly monotone.

Let $g \in\left(W_{0, \text { loc }}^{1, p(x)}(\Omega)\right)^{*}$, if $\langle g, \varphi\rangle \geq 0$, for all $\varphi \in W_{0, \text { loc }}^{1, p(x)}(\Omega), \varphi \geq 0$ a.e. in $\Omega$, then denote $g \geq 0$ in $\left(W_{0, \text { loc }}^{1, p(x)}(\Omega)\right)^{*}$; correspondingly, if $-g \geq 0$ in $\left(W_{0, \text { loc }}^{1, p(x)}(\Omega)\right)^{*}$, then denote $g \leq 0$ in $\left(W_{0, \text { loc }}^{1, p(x)}(\Omega)\right)^{*}$.

Definition 2.2. Let $u \in W_{\text {loc }}^{1, p(x)}(\Omega) \cap C(\Omega)$. If $A u \geq 0(A u \leq 0)$ in $\left(W_{0, \text { loc }}^{1, p(x)}(\Omega)\right)^{*}$, then $u$ is called a weak supersolution (weak subsolution) of $(\mathrm{P})$.

Copying the proof of [9], we have the following lemma.

Lemma 2.3 (comparison principle). Let $u, v \in W_{\text {loc }}^{1, p(x)}(\Omega) \cap C(\Omega)$ satisfy $A u-A v \geq 0$ in $\left(W_{0, \mathrm{loc}}^{1, p(x)}(\Omega)\right)^{*}$. Let $\varphi(x)=\min \{u(x)-v(x), 0\}$. If $\varphi(x) \in W_{0, \mathrm{loc}}^{1, p(x)}(\Omega)$ (i.e., $u \geq v$ on $\partial \Omega$ ), then $u \geq v$ a.e. in $\Omega$.

Lemma 2.4 (see [4, Theorem 1.1]). Under the conditions $\left(H_{1}\right)$ and $\left(H_{3}\right)$, if $u \in W^{1, p(x)}(\Omega)$ is a bounded weak solution of $-\Delta_{p(x)} u+e^{f(x, u)}=0$ in $\Omega$, then $u \in C_{\text {loc }}^{1, \vartheta}(\Omega)$, where $\vartheta \in(0,1)$ is a constant.

\section{Main results and proofs}

If $u$ is a radial solution of $(\mathrm{P})$, then $(\mathrm{P})$ can be transformed into

$$
\begin{gathered}
\left(r^{N-1}\left|u^{\prime}\right|^{p(r)-2} u^{\prime}\right)^{\prime}=r^{N-1} e^{f(r, u)}, \quad r \in(0, R), \\
u(0)=u_{0}, \quad u^{\prime}(0)=0, \quad u^{\prime}(r) \geq 0 \quad \text { for } 0<r<R .
\end{gathered}
$$

It means that $u(r)$ is increasing.

Theorem 3.1. If there exists a constant $\sigma \in[R / 2, R)$ such that

$$
f(r, u) \geq \alpha u^{s} \quad(\text { as } u \longrightarrow+\infty) \text { for } r \in[\sigma, R) \text { uniformly, }
$$

where $\alpha$ and $s$ are positive constants, then there exists a continuous function $\Phi_{1}(x)$ which satisfies $\Phi_{1}(x) \rightarrow+\infty($ as $d(x, \partial \Omega) \rightarrow 0)$, and such that, if $u$ is a weak solution of problem $(\mathrm{P})$, then $u(x) \leq$ $\Phi_{1}(x)$.

Proof. Let $R_{0} \in(\sigma, R)$. Denote

$$
\Theta(r, a, \lambda)=\int_{r}^{R_{0}}\left[\frac{a\left(a \ln \left(R-R_{0}-\lambda\right)^{-1}\right)^{1 / s-1}}{s\left(R-R_{0}-\lambda\right)}\right]^{\left(p\left(R_{o}\right)-1\right) /(p(t)-1)}\left[\frac{\left(R_{o}\right)^{N-1}}{t^{N-1}} \sin \varepsilon(t-\sigma)\right]^{1 /(p(t)-1)} d t .
$$

Define the function $g(r, a)$ on $[0, R)$ as

$$
g(r, a)= \begin{cases}\left(a \ln (R-r)^{-1}\right)^{1 / s}+k, & R_{0} \leq r<R \\ k-\Theta(r, a, 0)+\left(a \ln \left(R-R_{0}\right)^{-1}\right)^{1 / s}, & \sigma<r<R_{0} \\ k-\Theta(\sigma, a, 0)+\left(a \ln \left(R-R_{0}\right)^{-1}\right)^{1 / s}, & r \leq \sigma\end{cases}
$$


where $a>(1 / \alpha) \sup _{|x| \geq R_{0}} p(x)$ is a constant, $R_{0} \in(\sigma, R)$, and $R-R_{0}$ is small enough, $\varepsilon=\pi / 2\left(R_{0}-\sigma\right)$ and $k=\left(\left(2 p^{+} / \alpha\right) \ln \left(R-R_{0}\right)^{-1}\right)^{1 / s}+\Theta(\sigma, 2 a, 0)$.

Obviously, for any positive constant $a, g(r, a) \in C^{1}[0, R)$.

When $R_{0}<r<R$, we have

$$
\left(r^{N-1}\left|g^{\prime}\right|^{p(r)-2} g^{\prime}\right)^{\prime}=r^{N-1}\left(\frac{a^{1 / s}}{s}\right)^{p(r)-1} \frac{p(r)-1}{(R-r)^{p(r)}}\left(\ln (R-r)^{-1}\right)^{(1 / s-1)(p(r)-1)}(1+\Pi(r)),
$$

where

$$
\begin{aligned}
\Pi(r)= & \frac{(1 / s-1)}{\ln (R-r)^{-1}}+\frac{\left[r^{N-1}\left(a^{1 / s} / s\right)^{p(r)-1}\right]^{\prime}}{r^{N-1}\left(a^{1 / s} / s\right)^{p(r)-1}(p(r)-1)}(R-r) \\
& +\frac{-p^{\prime}(r) \ln (R-r)}{(p(r)-1)}(R-r)+\frac{(1 / s-1) p^{\prime}(r) \ln \ln (R-r)^{-1}}{(p(r)-1)}(R-r) .
\end{aligned}
$$

If $\left(R-R_{0}\right)$ is small enough, it is easy to see $|\Pi(r)| \leq 1 / 2$; from (3.5), we have

$$
\begin{aligned}
\left(r^{N-1}\left|g^{\prime}\right|^{p(r)-2} g^{\prime}\right)^{\prime} & \leq 2 r^{N-1}\left(\frac{a^{1 / s}}{s}\right)^{p(r)-1}(p(r)-1)(R-r)^{-p(r)}\left(\ln (R-r)^{-1}\right)^{(1 / s-1)(p(r)-1)} \\
& \leq r^{N-1}\left(\frac{1}{R-r}\right)^{\alpha a}=r^{N-1} e^{\alpha g^{s}} \leq r^{N-1} e^{f(r, g)}, \quad \forall r \in\left(R_{0}, R\right) .
\end{aligned}
$$

Obviously, if $R-R_{0}$ is small enough, then $g \geq\left(\left(2 p^{+} / \alpha\right) \ln \left(R-R_{0}\right)^{-1}\right)^{1 / s}$ is large enough, so we have

$$
\begin{aligned}
\left(r^{N-1}\left|g^{\prime}\right|^{p(r)-2} g^{\prime}\right)^{\prime} & =\varepsilon\left(R_{o}\right)^{N-1}\left[\frac{a\left(a \ln \left(R-R_{0}\right)^{-1}\right)^{1 / s-1}}{s\left(R-R_{0}\right)}\right]^{\left(p\left(R_{o}\right)-1\right)} \cos (\varepsilon(r-\sigma)) \\
& \leq r^{N-1} e^{\alpha g^{s}} \leq r^{N-1} e^{f(r, g)}, \quad \sigma<r<R_{0} .
\end{aligned}
$$

Obviously,

$$
\left(r^{N-1}\left|g^{\prime}\right|^{p(r)-2} g^{\prime}\right)^{\prime}=0 \leq r^{N-1} e^{f(r, g)}, \quad 0 \leq r<\sigma .
$$

Since $g(|x|, a)$ is a $C^{1}$ function on $B(0, R)$, if $0<R-R_{0}$ is small enough ( $R_{0}$ depends on $R, p, s, \alpha)$, from (3.7), (3.8), and (3.9), we can see that $g(|x|, a)$ is a supersolution of (P).

Define the function $g_{m}(r, a-\epsilon)$ on $[0, R-1 / m)$ as

$$
g_{m}(r, a-\epsilon)= \begin{cases}{\left[(a-\epsilon) \ln \left(R-\frac{1}{m}-r\right)^{-1}\right]^{1 / s}+k,} & R_{0} \leq r<R-\frac{1}{m}, \\ k-\Theta\left(r, a-\epsilon, \frac{1}{m}\right)+\left[(a-\epsilon) \ln \left(R-\frac{1}{m}-R_{0}\right)^{-1}\right]^{1 / s}, & \sigma<r<R_{0}, \\ k-\Theta\left(\sigma, a-\epsilon, \frac{1}{m}\right)+\left[(a-\epsilon) \ln \left(R-\frac{1}{m}-R_{0}\right)^{-1}\right]^{1 / s}, & r \leq \sigma,\end{cases}
$$


where $m$ is a big-enough integer such that $0<1 / m \leq\left(R-R_{0}\right) / 2, \varepsilon=\pi / 2\left(R_{0}-\sigma\right), 0<\epsilon<1$, is a positive small constant such that $\alpha(a-\epsilon)>\sup _{|x| \geq R_{0}} p(x)$.

Obviously, $g_{m}(|x|, a-\epsilon)$ is a supersolution of $(\mathrm{P})$ on $B(0, R-1 / m)$. If $u$ is a solution of $(\mathrm{P})$, according to the comparison principle, we get that $g_{m}(|x|, a-\epsilon) \geq u(x)$ for any $x \in B(0, R-1 / m)$. For any $x \in B(0, R-1 / m) \backslash B\left(0, R_{0}\right)$, we have $g_{m}(|x|, a-\epsilon) \geq g_{m+1}(|x|, a-\epsilon)$. Thus,

$$
u(x) \leq \lim _{m \rightarrow+\infty} g_{m}(|x|, a-\epsilon), \quad \forall x \in B(0, R) \backslash B\left(0, R_{0}\right) .
$$

When $d(x, \partial \Omega)>0$ is small enough, we have

$$
\lim _{m \rightarrow+\infty} g_{m}(|x|, a-\epsilon)<\left(a \ln (R-r)^{-1}\right)^{1 / s}+k \leq g(|x|, a) .
$$

According to the comparison principle, we obtain that $g(|x|, a) \geq u(x)$, for all $x \in B(0, R)$, then $\Phi_{1}(x)=g(|x|, a)$ is an upper control function of all of the solutions of $(\mathrm{P})$. The proof is completed.

Theorem 3.2. If there exists a $\sigma \in[R / 2, R)$ such that

$$
f(r, u) \leq \beta u^{s} \quad(\text { as } u \longrightarrow+\infty) \text { for } r \in[\sigma, R) \text { uniformly, }
$$

where $\beta$ and $s$ are positive constants, then there exists a continuous function $\Phi_{2}(x)$ which satisfies $\Phi_{2}(x) \rightarrow+\infty$ (as $\left.d(x, \partial \Omega) \rightarrow 0\right)$, and such that, if $u(x)$ is a solution of problem $(\mathrm{P})$, then $u(x) \geq$ $\Phi_{2}(x)$.

Proof. Let $z_{1}$ be a radial solution of

$$
-\Delta_{p(x)} z_{1}(x)=-\mu \text { in } \Omega_{1}=B(0, \sigma), z_{1}=0 \text { on } \partial \Omega_{1}
$$

where $\mu>2$ is a positive constant. We denote $z_{1}=z_{1}(r)=z_{1}(|x|)$, then $z_{1}$ satisfies $z_{1}(\sigma)=0$, $z_{1}^{\prime}(0)=0$, and

$$
z_{1}^{\prime}=\left|\frac{r \mu}{N}\right|^{1 /(p(r)-1)}, \quad z_{1}=-\int_{r}^{\sigma}\left|\frac{r \mu}{N}\right|^{1 /(p(r)-1)} d r .
$$

Denote $h_{b}(r, \delta)$ on $\left[\sigma, R_{0}\right]$ as

$$
\begin{aligned}
h_{b}(r, \delta)=\int_{r}^{R_{0}}\{ & \frac{\left(R_{o}\right)^{N-1}}{t^{N-1}} \frac{t-\sigma}{R_{0}-\sigma}\left[\frac{b\left(b \ln \left(R+\delta-R_{0}\right)^{-1}\right)^{1 / s-1}}{s\left(R+\delta-R_{0}\right)}\right]^{p\left(R_{o}\right)-1} \\
& \left.+\frac{(\sigma)^{N-1}}{t^{N-1}} \frac{R_{0}-t}{R_{0}-\sigma}\left[\left|\frac{t \mu}{N}\right|^{1 /(p(t)-1)}\right]^{p(\sigma)-1}\right\}^{1 /(p(t)-1)} d t
\end{aligned}
$$

It is easy to see that

$$
-h_{b}^{\prime}(\sigma, 0)=z_{1}^{\prime}(\sigma)=\left|\frac{\sigma \mu}{N}\right|^{1 /(p(\sigma)-1)}, \quad-h_{b}^{\prime}\left(R_{0}, 0\right)=\frac{b\left(b \ln \left(R-R_{0}\right)^{-1}\right)^{1 / s-1}}{s\left(R-R_{0}\right)} .
$$


Define the function $v(r, b)$ on $B(0, R)$ as

$$
v(r, b)= \begin{cases}\left(b \ln (R-r)^{-1}\right)^{1 / s}-k^{*}, & R_{0} \leq r<R, \\ \left(b \ln \left(R-R_{0}\right)^{-1}\right)^{1 / s}-k^{*}-h_{b}(r, 0), & \sigma<r<R_{0}, \\ -\int_{r}^{\sigma}\left|\frac{r \mu}{N}\right|^{1 /(p(r)-1)} d r+\left(b \ln \left(R-R_{0}\right)^{-1}\right)^{1 / s}-k^{*}-h_{b}(\sigma, 0), & r \leq \sigma,\end{cases}
$$

where $b \in\left(0,(1 / \beta) \inf _{|x| \geq R_{0}} p(x)\right)$ is a constant, $R_{0} \in(\sigma, R)$, and $R-R_{0}$ is small enough, and $k^{*}=\left(\left(2 p^{+} / \beta\right) \ln 2\left(R-R_{0}\right)^{-1}\right)^{1 / s}$.

Obviously, for any positive constant $b, v(r, b) \in C^{1}[0, R)$.

Similar to the proof of Theorem 3.1, when $R-R_{0}$ is small enough, we have

$$
\left(r^{N-1}\left|v^{\prime}\right|^{p(r)-2} v^{\prime}\right)^{\prime} \geq r^{N-1} e^{f(r, v)}, \quad \forall r \in\left(R_{0}, R\right) .
$$

When $R-R_{0}$ is small enough, for all $r \in\left(\sigma, R_{0}\right)$, since $f(r, v) \leq 0$, then

$$
\left(r^{N-1}\left|v^{\prime}\right|^{p(r)-2} v^{\prime}\right)^{\prime} \geq \frac{1}{2} \frac{\left(R_{o}\right)^{N-1}}{R_{0}-\sigma}\left[\frac{b\left(b \ln \left(R-R_{0}\right)^{-1}\right)^{1 / s-1}}{s\left(R-R_{0}\right)}\right]^{p\left(R_{0}\right)-1} \geq r^{N-1} e^{f(r, v)} .
$$

Obviously,

$$
\left(r^{N-1}\left|v^{\prime}\right|^{p(r)-2} v^{\prime}\right)^{\prime}=r^{N-1} \mu \geq r^{N-1} e^{f(r, v)}, \quad \forall r \in(0, \sigma) .
$$

Combining (3.19), (3.20), and (3.21), we can see that $v(r, a)$ is a subsolution of (P).

Define the function $v_{m}(r, b+\epsilon)$ on $B(0, R)$ as

$$
v_{m}(r, b+\epsilon)= \begin{cases}{\left[(b+\epsilon) \ln \left(R+\frac{1}{m}-r\right)^{-1}\right]^{1 / s}-k^{*},} & R_{0} \leq r<R, \\ {\left[(b+\epsilon) \ln \left(R+\frac{1}{m}-R_{0}\right)^{-1}\right]^{1 / s}-k^{*}-h_{b+\epsilon}\left(r, \frac{1}{m}\right),} & \sigma<r<R_{0}, \\ -\int_{r}^{\sigma}\left|\frac{\mu r}{N}\right|^{1 /(p(r)-1)} d r+\left[(b+\epsilon) \ln \left(R+\frac{1}{m}-R_{0}\right)^{-1}\right]^{1 / s}-k^{*}-h_{b+\epsilon}\left(\sigma, \frac{1}{m}\right), & r \leq \sigma,\end{cases}
$$

where $\epsilon$ is a small-enough positive constant such that $(b+\epsilon)<(1 / \beta) \inf _{|x| \geq R_{0}} p(x)$.

We can see that $v_{m}(r, b+\epsilon) \in C^{1}([0, R))$ is a subsolution of $(\mathrm{P})$ on $B\left(R_{0}, R\right)$, according to the comparison principle, we get that $v_{m}(|x|, b+\epsilon) \leq u(x)$ for any $x \in B(0, R)$. For any $x \in B(0, R) \backslash B\left(0, R_{0}\right)$, we have $v_{m}(|x|, b+\epsilon) \leq v_{m+1}(|x|, b+\epsilon)$. Thus,

$$
u(x) \geq \lim _{m \rightarrow+\infty} v_{m}(|x|, b+\epsilon), \quad \forall x \in B(0, R) \backslash B\left(0, R_{0}\right) .
$$

When $d(x, \partial \Omega)$ is small enough, we have

$$
\lim _{m \rightarrow+\infty} v_{m}(|x|, b+\epsilon)>v(|x|, b) .
$$

From the comparison principle, we obtain $v(|x|, b) \leq u(x), \forall x \in B(0, R)$, then $\Phi_{2}(x)=$ $v(|x|, b)$ is a lower control function of all of the solutions of $(\mathrm{P})$. 
Theorem 3.3. If $\inf _{x \in \Omega} p(x)>N$ and there exists a $\sigma \in[R / 2, R)$ such that

$$
f(r, u) \geq a u^{s} \quad(\text { as } u \longrightarrow+\infty) \text { for } r \in[\sigma, R) \text { uniformly, }
$$

where $a$ and s are positive constants, then $(\mathrm{P})$ possesses a solution.

Proof. In order to deal with the existence of boundary blow-up solutions of $(\mathrm{P})$, let us consider the problem

$$
\begin{gathered}
-\Delta_{p(x)} u+e^{f(x, u)}=0 \quad \text { in } \Omega, \\
u(x)=j \quad \text { for } x \in \partial \Omega,
\end{gathered}
$$

where $j=1,2, \ldots$ Since $\inf _{x \in \Omega} p(x)>N$, then $W^{1, p(x)}(\Omega) \hookrightarrow C^{\alpha}(\bar{\Omega})$, where $\alpha \in(0,1)$. The relative functional of (3.26) is

$$
\varphi(u)=\int_{\Omega} \frac{1}{p(x)}|\nabla u(x)|^{p(x)} d x+\int_{\Omega} F(x, u) d x,
$$

where $F(x, u)=\int_{0}^{u} e^{f(x, t)} d t$. Since $\varphi$ is coercive in $X_{j}:=j+W_{0}^{1, p(x)}(\Omega)$, then $\varphi$ possesses a nontrivial minimum point $u_{j}$, then problem (3.26) possesses a weak solution $u_{j}$. According to the comparison principle, we get $u_{j}(x) \leq u_{j+1}(x)$ for any $x \in \Omega$ and $j=1,2, \ldots$ Since $\Phi_{1}(x)$ defined in Theorem 3.1 is a supersolution, according to the comparison principle, we have $u_{j}(x) \leq \Phi_{1}(x)$ on $\Omega$ for all $j=1,2, \ldots$. Since $\Phi_{1}(x)$ is locally bounded, from Lemma 2.4, every weak solution of $(\mathrm{P})$ is a locally $C_{\text {loc }}^{1, \vartheta}$ function. Thus, $\left\{u_{j}(x)\right\}$ possesses a subsequence (we still denote it by $\left.\left\{u_{j}(x)\right\}\right)$, such that $\lim _{j \rightarrow \infty} u_{j}=u$ is a solution of $(\mathrm{P})$.

\section{Acknowledgments}

This work was supported by the National Science Foundation of China (10701066 \& 10671084)and China Postdoctoral Science Foundation (20070421107) and the Natural Science Foundation of Henan Education Committee (2007110037).

\section{References}

[1] Y. Chen, S. Levine, and M. Rao, "Variable exponent, linear growth functionals in image restoration," SIAM Journal on Applied Mathematics, vol. 66, no. 4, pp. 1383-1406, 2006.

[2] M. Rúzicka, Electrorheological Fluids: Modeling and Mathematical Theory, vol. 1748 of Lecture Notes in Mathematics, Springer, Berlin, Germany, 2000.

[3] X.-L. Fan and D. Zhao, "On the spaces $L^{p(x)}(\Omega)$ and $W^{m, p(x)}(\Omega)$," Journal of Mathematical Analysis and Applications, vol. 263, no. 2, pp. 424-446, 2001.

[4] X.-L. Fan, "Global $C^{1, \alpha}$ regularity for variable exponent elliptic equations in divergence form," Journal of Differential Equations, vol. 235, no. 2, pp. 397-417, 2007.

[5] X.-L. Fan and Q. Zhang, "Existence of solutions for $p(x)$-Laplacian Dirichlet problem," Nonlinear Analysis: Theory, Methods \& Applications, vol. 52, no. 8, pp. 1843-1852, 2003.

[6] X.-L. Fan, Q. Zhang, and D. Zhao, "Eigenvalues of $p(x)$-Laplacian Dirichlet problem," Journal of Mathematical Analysis and Applications, vol. 302, no. 2, pp. 306-317, 2005.

[7] O. Kováčik and J. Rákosník, “On spaces $L^{p(x)}(\Omega)$ and $W^{k, p(x)}(\Omega)$, , Czechoslovak Mathematical Journal, vol. 41(116), no. 4, pp. 592-618, 1991. 
[8] S. G. Samko, "Density $C_{0}^{\infty}\left(\mathbb{R}^{N}\right)$ in the generalized Sobolev spaces $W^{m, p(x)}\left(\mathbb{R}^{N}\right)$," Doklady Rossiǔskaya Akademiya Nauk, vol. 369, no. 4, pp. 451-454, 1999.

[9] Q. Zhang, "A strong maximum principle for differential equations with non standard $p(x)$-growth conditions," Journal of Mathematical Analysis and Applications, vol. 312, no. 1, pp. 24-32, 2005.

[10] Q. Zhang, "Existence of positive solutions for elliptic systems with non standard $p(x)$-growth conditions via sub-supersolution method," Nonlinear Analysis: Theory, Methods \& Applications, vol. 67, no. 4, pp. 1055-1067, 2007.

[11] Q. Zhang, "Existence and asymptotic behavior of blow-up solutions to a class of $p(x)$-Laplacian problems," Journal of Mathematical Analysis and Applications, vol. 329, no. 1, pp. 472-482, 2007.

[12] Q. Zhang, "Existence of positive solutions for a class of $p(x)$-Laplacian systems," Journal of Mathematical Analysis and Applications, vol. 333, no. 2, pp. 591-603, 2007.

[13] Q. Zhang, "The asymptotic behavior of solutions for $p(x)$-Laplace equations," Journal of Zhengzhou University of Light Industry, vol. 24, no. 5, pp. 92-95, 2007.

[14] Q. Zhang, "Oscillatory property of solutions for $p(t)$-Laplacian equations," Journal of Inequalities and Applications, vol. 2007, Article ID 58548, 8 pages, 2007.

[15] Q. Zhang, "Existence and asymptotic behavior of positive solutions to $p(x)$-Laplacian equations with singular nonlinearities," Journal of Inequalities and Applications, vol. 2007, Article ID 19349, 9 pages, 2007.

[16] J. B. Keller, "On solutions of $\Delta u=f(u)$, " Communications on Pure and Applied Mathematics, vol. 10, pp. 503-510, 1957.

[17] A. V. Lair, "A necessary and sufficient condition for existence of large solutions to semilinear elliptic equations," Journal of Mathematical Analysis and Applications, vol. 240, no. 1, pp. 205-218, 1999.

[18] A. Mohammed, "Existence and asymptotic behavior of blow-up solutions to weighted quasilinear equations," Journal of Mathematical Analysis and Applications, vol. 298, no. 2, pp. 621-637, 2004. 